\title{
Transverse dipole spin modes in quantum dots
}

\author{
E. Lipparini and M. Barranco* \\ Dipartimento di Fisica, Università di Trento, I-38050 Povo, Italy \\ A. Emperador and M. Pi \\ Departament d'Estructura i Constituents de la Materia, Facultat de Física, Universitat de Barcelona, E-08028 Barcelona, Spain \\ Ll. Serra \\ Departament de Física, Universitat de les Illes Balears, E-07071 Palma de Mallorca, Spain
}

(Received 23 March 1999)

\begin{abstract}
We have carried out a systematic analysis of the transverse dipole spin response of a large-size quantum dot within time-dependent current density functional theory. Results for magnetic fields corresponding to integer filling factors are reported, as well as a comparison with the longitudinal dipole spin response. As in the two-dimensional electron gas, the spin response at high-spin magnetization is dominated by a low-energy transverse mode. [S0163-1829(99)14435-7]
\end{abstract}

\section{INTRODUCTION}

Resonant inelastic light scattering has become a very useful tool to study quantum dot (QD) excitations. ${ }^{1-4}$ It had been extensively employed before to study electron single-layers and quantum wires in semiconductor heterostructures (see for example Refs. 5 and 6), and currently it is also being applied to study electron multilayers. ${ }^{7,8}$ We refer the reader to Refs. 9 and 10 for a review of this experimental technique.

A major advantage of resonant inelastic light scattering over far infrared (FIR) optical absorption techniques used in the past ${ }^{11,12}$ is that the former allows to disentangle and identify, using polarization selection rules in the backwards geometry, ${ }^{9}$ charge density (CDE), spin density (SDE), and single-particle (SPE) electron excitations in the same sample, whereas FIR absorption is only sensitive to charge-density excitations. In inelastic light scattering, when the polarizations of the incoming and scattered photon are parallel (polarized geometry) CDE's are observed, whereas when the polarizations are perpendicular (depolarized geometry) SDE's dominate the spectrum. This is due to the structure of the scattering cross section, which besides the charge or spin electronic strength function contains the scalar or vector product of the photon polarizations, respectively. ${ }^{13,14}$ Yet, CDE's are seen with some intensity in the depolarized spectrum. ${ }^{4}$ SPE's are mostly detected under conditions of extreme resonance, whereas CDE's and SDE's can be observed at incident photon energies far above the effective band gap. ${ }^{3}$ This helps disentangle SDE's from SPE's, which are in the same energy range at small spin magnetizations.

For a QD in the $x y$ plane submitted to a static magnetic field $B$ in the $z$ direction, SDE's may involve electronic spin flips or not. The later excitations are referred to as longitudinal SDE's, and the former as transverse SDE's. This means that SDE's are caused by one-electron excitation operators of the kind

$$
\begin{gathered}
F_{z}=\sum_{i} f\left(\vec{r}_{i}\right) \sigma_{z}^{i} \text { longitudinal }\left(\Delta S_{z}=0\right), \\
F_{ \pm}=\sum_{i} f\left(\vec{r}_{i}\right) \sigma_{ \pm}^{i} \text { transverse }\left(\Delta S_{z}= \pm 1\right),
\end{gathered}
$$

where $S_{z}$ is the $z$ component of the spin of the dot, and $\sigma_{z}$ is the $z$ component of the Pauli matrix vector. $\sigma_{ \pm}=\left(\sigma_{x}\right.$ $\left.\pm \imath \sigma_{y}\right) / 2$ are the spin-flip operators

$$
\sigma_{+}|\uparrow\rangle=\sigma_{-}|\downarrow\rangle=0 ; \quad \sigma_{+}|\downarrow\rangle=|\uparrow\rangle ; \quad \sigma_{-}|\uparrow\rangle=|\downarrow\rangle .
$$

Due to rotational invariance in spin space, longitudinal and transverse SDE's are degenerated at $B=0$ if $S_{z}=0$ in the ground state (gs). When the magnetic field is not zero, rotational invariance is broken by the Zeeman term. Then if the gs is almost paramagnetic, i.e., $S_{z} \sim 0$ as it occurs at even filling factors $\nu$, the SDE's are expected to split in a simple way:

$$
\omega_{ \pm}^{t r}=\omega^{l o n} \pm g^{*} \mu_{B} B
$$

where the superscript lon (tr) indicates the longitudinal(transverse) character of the mode, and the subscript \pm corresponds to the two possible spin-flip transitions. $g^{*}$ is the effective gyromagnetic factor and $\mu_{B}$ is the Bohr magneton. A thorough discussion of longitudinal dipole SDE's in quantum dots within time-dependent local-spin density theory (TDLSDT) has been presented in Refs. 15 and 16. If the gs has a large $S_{z}$, i.e., a large spin magnetization (ferromagnetic gs), as it happens at odd filling factors $\nu$, Eq. (3) no longer holds and longitudinal and transverse SDE's display dramatic differences arising from the spin dependence of the electron-hole (e-h) vertex corrections.

To study transverse dipole spin modes we have resorted to current density-functional theory ${ }^{17,18}$ (CDFT) together with its time-dependent version (TDCDFT) ${ }^{19}$ For the physics we aim to describe here, local-spin density theory (LSDT) and CDFT sensibly yield the same results. CDFT is expected to be more reliable than LSDT at high-magnetic fields,$^{20}$ but the residual interaction in the longitudinal spin 
channel is very cumbersome to work out within TDCDFT. For this reason, whenever we have had to work out the longitudinal spin response for the sake of comparison with the transverse one, we have resorted to TDLSDT. We will see that the spin dipole response is dominated by the transverse component, especially for filling factors smaller than 2. In the following we shall use the terms density-functional theory (DFT) and time-dependent density-functional theory (TDDFT) when the statement applies to either version of the general method.

The spin and density response of the two-dimensional electron gas (2DEG) has been thoroughly studied by Kallin and Halperin ${ }^{21}$ and MacDonald. ${ }^{22}$ As these authors, we have mainly addressed the response of quantum dots corresponding to integer filling factor gs's. We have considered only three cases whose ground state is the finite size analogue of a partially filled 2DEG configuration between $\nu=1$ and 2 . One should regard these results as qualitative, since the corresponding ground states are believed to be very complicated, strongly correlated ones, and the use of TDDFT to describe them may be questioned. Nonetheless, these results display the gross trends of the excitation spectrum, and for that reason we have considered them here.

\section{RESULTS}

We have taken as a case of study a circularly symmetric QD of radius $R_{d o t} \sim 164 \mathrm{~nm}$ made of $N=210$ electrons in a GaAs- $\mathrm{Al}_{x} \mathrm{Ga}_{1-x} \mathrm{As}$ heterostructure. The gs of this dot has been described in detail in Ref. 18. Throughout this paper, we shall be using effective atomic units, whose definition and value for GaAs can be found for example in that reference.

A major advantage of considering a rather large QD for the present discussion is that several integer filling factor gs configurations can be identified as a function of $B$. This allows to discuss the influence of the paramagnetic or ferromagnetic character of the gs on the excitation spectrum. The large number of relatively close single particle ( $\mathrm{sp}$ ) levels that one has to handle to obtain the gs and strength functions poses some technical problems, and is the token to pay for microscopically study large QD. In this paper, we have used a small temperature $T \leqslant 0.1 \mathrm{~K}$. This facilitates the calculations while it does not introduce any appreciable thermal effect in the results.

In the following, we limit the analysis to the most interesting dipole mode, for which the $f\left(\vec{r}_{i}\right)$ in Eq. (1) is $x_{i}$. Furthermore, to take advantage of the imposed circular symmetry, we have considered as dipole operators the following ones:

$$
\begin{gathered}
D_{ \pm 1,0}=\frac{1}{2} \sum_{j} r_{j} e^{ \pm \imath \theta_{j}} \sigma_{z}^{j}\left(\Delta S_{z}=0\right), \\
D_{ \pm 1, \pm}=\frac{1}{2} \sum_{j} r_{j} e^{ \pm \imath \theta_{j}} \sigma_{ \pm}^{j}\left(\Delta S_{z}= \pm 1\right),
\end{gathered}
$$

as well as the combinations:

$$
\sum_{j} x^{j} \sigma_{z}^{j}=D_{+1,0}+D_{-1,0} \quad\left(\Delta S_{z}=0\right)
$$

$$
\sum_{j} x^{j} \sigma_{ \pm}^{j}=D_{+1, \pm}+D_{-1, \pm}\left(\Delta S_{z}= \pm 1\right),
$$

where the subscript \pm 1 represents the orbital angular momentum carried by the excitation, each one corresponding to a different kind of left- or right-circularly polarized light. Within TDDFT, the external probe represented by these operators has also a harmonic time dependence of frequency $\omega$.

The method we have used to obtain the spin response has been described in Ref. 19 for the transverse case, and in Refs. 15 and 16 for the longitudinal one, so we do not need to repeat it here. We want to recall that the mean field entering the Kohn-Sham (KS) equations changes due to the dynamical spin magnetization induced by the external field. Within TDDFT, it is assumed that electrons respond as free particles to the sum of the external plus induced field, which leads to a Dyson-type integral equation for the correlation function $\chi$

$$
\begin{aligned}
\chi\left(\vec{r}, \vec{r}^{\prime}, \omega\right)= & \chi^{0}\left(\vec{r}, \vec{r}^{\prime}, \omega\right)+\int d \vec{r}_{1} d \vec{r}_{2} \chi^{0}\left(\vec{r}, \vec{r}_{1}, \omega\right) \\
& \times V_{e h}\left(\vec{r}_{1}, \vec{r}_{2}\right) \chi\left(\vec{r}_{2}, \vec{r}^{\prime}, \omega\right),
\end{aligned}
$$

where $\chi^{0}$ is the free electron correlation function and $V_{e h}$ is the residual e-h interaction. From $\chi$ it is possible to obtain the induced spin magnetization corresponding to any of the above excitation operators and then determine the dynamical polarizability function $\alpha(\omega)$. Finally, the strength function $S(\omega)$ is obtained from the imaginary part of the dynamical polarizability function as $S(\omega)=1 / \pi \operatorname{Im}[\alpha(\omega)]$. This procedure has been explicited in Ref. 19 for the $D_{+1,-}$ operator. All these functions can be labeled according to the $\Delta L_{z}$ $= \pm 1$ and $\Delta S_{z}=0, \pm 1$ changes induced in the excitation process. In the following, we shall analyze the strength functions corresponding to the operators in Eq. (5), which we call $x \sigma_{+}, x \sigma_{-}, x \sigma_{z}$, and $x \sigma_{x}=x\left(\sigma_{+}+\sigma_{-}\right)$.

Some characteristics of the strength functions are easily understood in terms of the uncorrelated $\mathrm{e}-\mathrm{h}$ excitations (SPE's) used to build the corresponding $\chi^{0}(\omega)$, whose basic ingredients are the KS single-particle energies $\epsilon_{n l \sigma}$ and wave functions $\varphi_{n l \sigma}(\vec{r})$ obtained from the solution of the $\mathrm{KS}$ equations that within LSDT read $^{16}$

$$
\begin{aligned}
& {\left[-\frac{1}{2} \nabla^{2}+\frac{1}{2} \omega_{c} \ell_{z}+\frac{1}{8} \omega_{c}^{2} r^{2}+V^{+}(r)+V^{H}+V^{x c}\right.} \\
& \left.\quad+\left(W^{x c}+\frac{1}{2} g^{*} \mu_{B} B\right) \sigma_{z}\right] \varphi_{n l \sigma}(\vec{r})=\epsilon_{n l \sigma} \varphi_{n l \sigma}(\vec{r}),
\end{aligned}
$$

where $V^{+}(r)$ is the confining potential and $V^{H}$ $=\int d \vec{r} \rho\left(\vec{r}^{\prime}\right) /\left|\vec{r}-\vec{r}^{\prime}\right|$ is the Hartree potential. $V^{x c}$ $=\partial \mathcal{E}_{x c}(\rho, m) /\left.\partial \rho\right|_{g s}$ and $W^{x c}=\partial \mathcal{E}_{x c}(\rho, m) /\left.\partial m\right|_{g s}$ are, respectively, the spin-independent and spin-dependent exchangecorrelation sp potentials obtained from the exchangecorrelation energy density $\mathcal{E}_{x c}(\rho, m)$, and $\rho$ and $m$ are the electron and spin magnetization densities $\rho(\vec{r}) \equiv \rho^{\uparrow}(\vec{r})$ $+\rho^{\downarrow}(\vec{r}), m(\vec{r}) \equiv \rho^{\uparrow}(\vec{r})-\rho^{\downarrow}(\vec{r}) . \quad \mathcal{E}_{x c}(\rho, m)$ has been constructed as indicated in Ref. 16. Within CDFT, the KS equations are more cumbersome to write down. ${ }^{17}$ Still, they have a similar structure, and what one has to keep in mind for the discussions that follow is the existence of an exchange- 

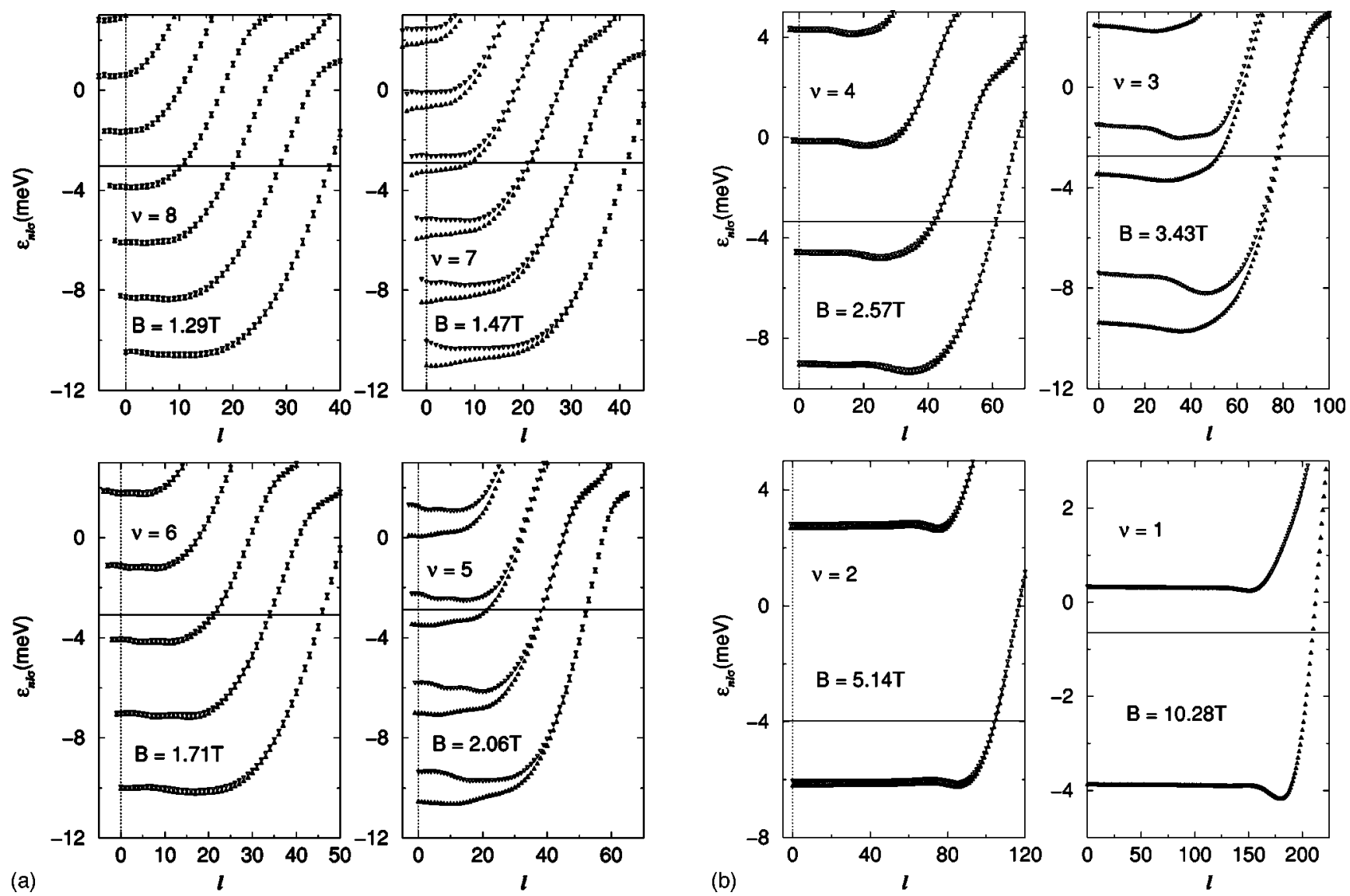

FIG. 1. (a) Single-particle energies as a function of orbital angular momentum $l$ for $\nu=8$ to 5 . The horizontal solid lines represent the electron chemical potential. The full, upright triangles represent $(M, \uparrow)$ bands, and the empty, downright triangles represent $(M, \downarrow)$ bands. (b) Same as (a) for $\nu=4$ to 1 .

correlation potential, which plays the role of $V^{x c}$, and another exchange-correlation potential, which plays the role of $W^{x c}$ in the above equation.

One should be aware that the residual e-h interaction may change drastically the picture extracted from the uncorrelated e-h excitations. Yet, as a useful guide for the discussion, we collect in Fig. 1 the sp energies as a function of the angular momentum $l$ for $\nu=8$ to 1 , corresponding to $B$ values from 1.29 to $10.28 \mathrm{~T}$. In the bulk of the dot (small $l$ values), these sp energies bear the band characteristics of the 2DEG, having similar $\epsilon_{n l \sigma}$ the sp states that have the same $s_{z}$ and yield the same Landau level index $M \equiv n+(|l|-l) / 2$, where $n$ is the sp radial quantum number. For this dot, the filling factor $\nu$ does represent the number of occupied Landau bands, each one labeled as $(M, \uparrow)$ or $(M, \downarrow)$. In Fig. 1, upright full triangles represent $s_{z}=\uparrow$ sp states, and downright, empty triangles, $s_{z}=\downarrow \mathrm{sp}$ states. The horizontal lines represent the electron chemical potential. All the occupied bands are shown, but to build the valence space of sp states for calculating the correlation functions we have usually considered more empty states than those shown in the figure.

It is worth to notice the small energy difference between the $(M, \uparrow)$ and $(M, \downarrow)$ bands for even $\nu$ values $\Delta E_{\downarrow} \uparrow$ $\sim\left|g^{*} \mu_{B} B\right|$, which has its origin in the Zeeman term, as compared to the large energy difference between the same bands if $\nu$ is odd, even if the applied $B$ is relatively small; compare for instance the $\nu=7$ and 6 cases. That difference in ferromagnetic gs's mostly comes from the spin-dependent exchange-correlation potential $\Delta E_{\downarrow \uparrow} \sim 2\left|W^{x c}\right|$, which is zero or very small in paramagnetic gs's, and sizeable in ferromagnetic gs's, largely overcoming the Zeeman energy. Hartree-Fock $^{23}$ (HF) and LSDT or CDFT (Ref. 17) yield such large gaps, whereas the Hartree approximation does not. The role of electron-electron interactions in producing these gaps when $\nu$ is odd has been stressed in Ref. 24. We are going to see that the effect of the gap is paramount on the transverse spin response.

\section{A. Strength functions}

Figure 2 shows the strength function $S(\omega)$ (solid lines) corresponding to $x \sigma_{x}$. For the sake of clarity, we have decomposed $x \sigma_{x}$ into its $x \sigma_{+}$and $x \sigma_{-}$components, which are drawn in Figs. 3 and 4, respectively. The associated free responses, i.e., SPE's, are represented by dashed lines. The functions are given in effective atomic units, and the frequencies in meV.

Let us first comment on the results corresponding to paramagnetic gs's in which both spin up and down sp states have a tendency to be equally populated yielding a small $S_{z}$ value. As a consequence, the attractive e-h residual interaction is weak, and SDE's and SPE's lie in the same energy range. In 


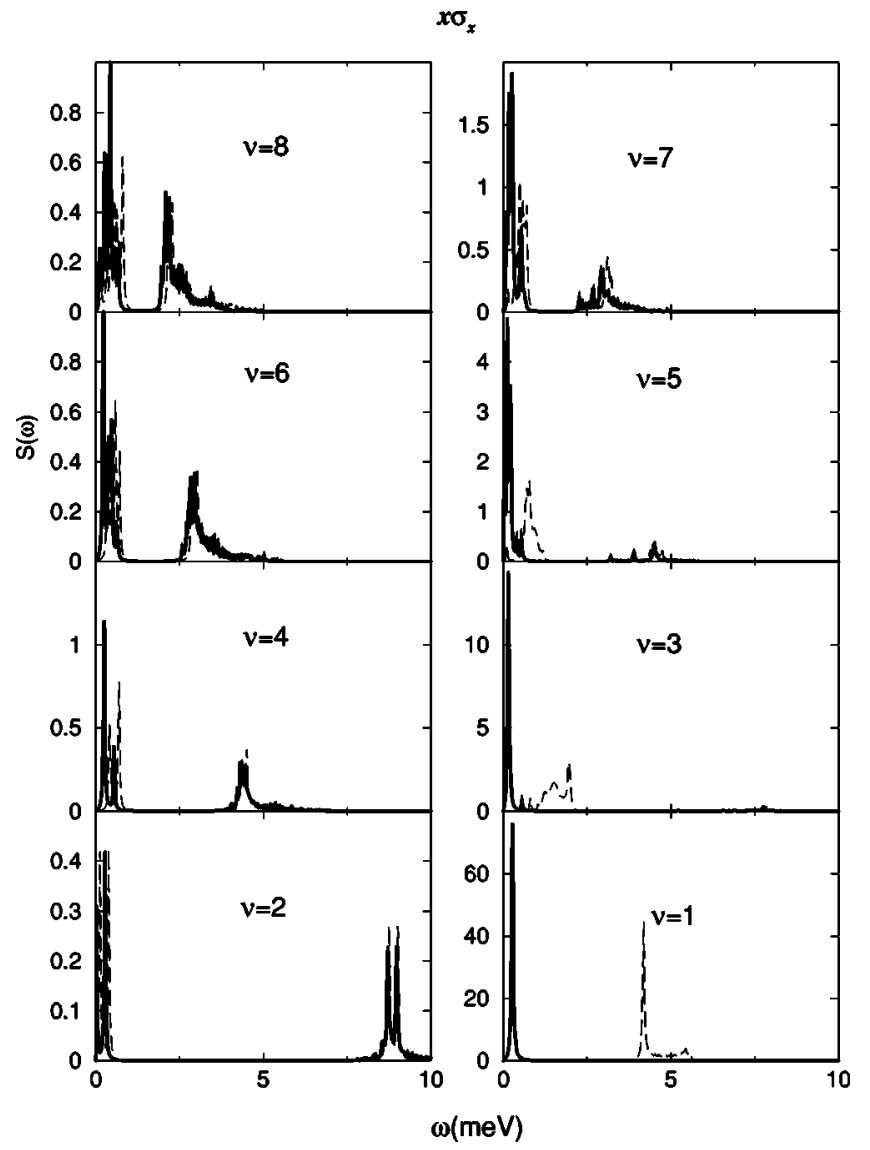

FIG. 2. Strength function corresponding to $x \sigma_{x}$ (solid lines). The dashed lines represent the free strength function. $S(\omega)$ is in effective atomic units divided by $10^{5}$.

both $x \sigma_{+}$and $x \sigma_{-}$components, the strength displays a high energy structure with a frequency always close to the free strength (see also Fig. 5), and a low-energy structure. For paramagnetic gs's, the low-energy excitation is a transverse spin edge mode built from e-h pairs near the Fermi level. These pairs can be easily identified in Figs. 1(a) and 1(b), as they are at the intersection of the chemical potential with the Landau bands. The sp band structure also explains why the edge mode is more fragmented at low magnetic fields. For example, at $\nu=8$ four e-h pairs, each one involving quite different sp orbital angular momenta from the other pairs, are contributing to the $x \sigma_{-}$strength, whereas only one pair is contributing at $\nu=2$. These pairs are weakly correlated among them and the result is an edge mode fourfold fragmented at $\nu=8$, threefold fragmented at $\nu=6$, and so on. This nicely corresponds to the number of crossings of the Fermi level with the $(M, \uparrow \downarrow)$ bands in Fig. 1 .

In the case of $x \sigma_{+}$, the edge mode is less fragmented because some spin-flip e-h transitions having $\Delta S_{z}=+1$ are Pauli blocked by our arbitrary election of $B$ in the positive direction of the $z$ axis (we recall that one has to have $\Delta L_{z}$ $= \pm 1$, which cannot always be fulfilled simultaneously with the spin and edge conditions). The lacking of the edge state in the $\nu=4$ case is due to the particular sp structure around the Fermi level at $B=2.57 \mathrm{~T}$. This accidental fact has no relevance for the general discussion.

As anticipated, the e-h residual interaction produces a dramatic effect when the gs is ferromagnetic. Even for moder-

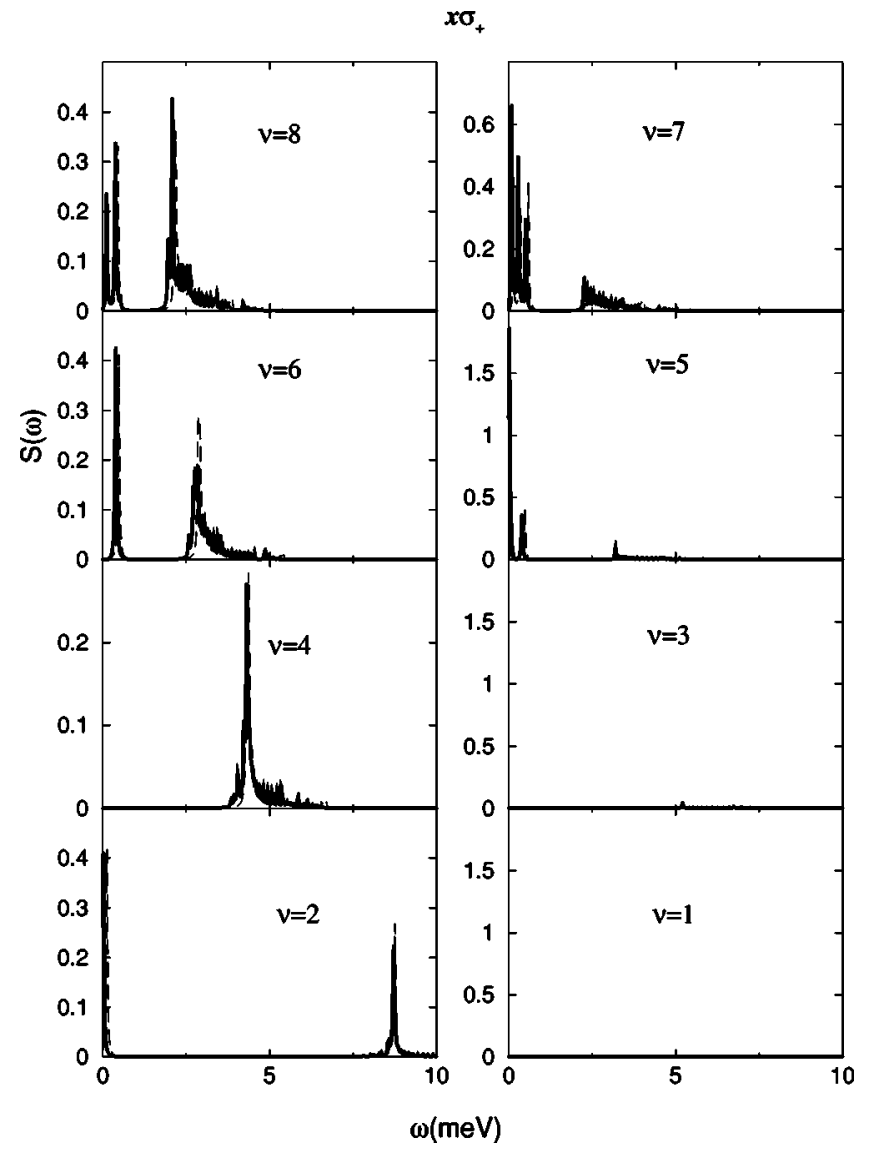

FIG. 3. Strength function corresponding to $x \sigma_{+}$(solid lines). The dashed lines represent the free strength function. $S(\omega)$ is in effective atomic units divided by $10^{5}$.

ately intense magnetic fields, the transverse spin mode emerges in these gs's as a very collective, undamped excitation whose energy we will see in the next subsection depends little on the size of the dot provided it is of the present size or larger. This is somehow the analogous of generalized Kohn's theorem $^{25,26}$ for charge density excitations in a QD parabolically confined, and it has the same physical origin, namely, the exact (or nearly exact in the spin case) cancelation between the bare and induced e-h interactions.

It can be seen from Fig. 3 that when the dot is fully polarized at $\nu=1, x \sigma_{+}$no longer excites it because of Pauli blocking. Even at $\nu=3(B=3.43 \mathrm{~T})$, its strength is very small. On the contrary, the excitation produced by $x \sigma_{-}$displayed in Fig. 4 is appreciably redshifted from the free response. The difference between both situations reveals the strength of vertex corrections arising from exchangecorrelation terms of the electron-electron interaction, which within TDDFT (Refs. 19 and 16) are the only ones contributing to dress the free e-h vertex in the spin channel. This effect is more sizeable at $\nu=1$ when the system is fully polarized.

The low-energy peak excited by $x \sigma_{-}$is taking almost all the dipole strength, as we will show later. Low-energy SDE's in ferromagnetic gs's caused either by $x \sigma_{+}$or $x \sigma_{-}$ are not edge, but bulk spin excitations. Again, Fig. 1 helps understanding this. For these odd $\nu$ gs's, the Fermi level is between the $\left(M_{\max }, \uparrow\right)$ and $\left(M_{\max }, \downarrow\right)$ Landau bands, the former being occupied and the later empty. Although finite 
$x \sigma_{-}$

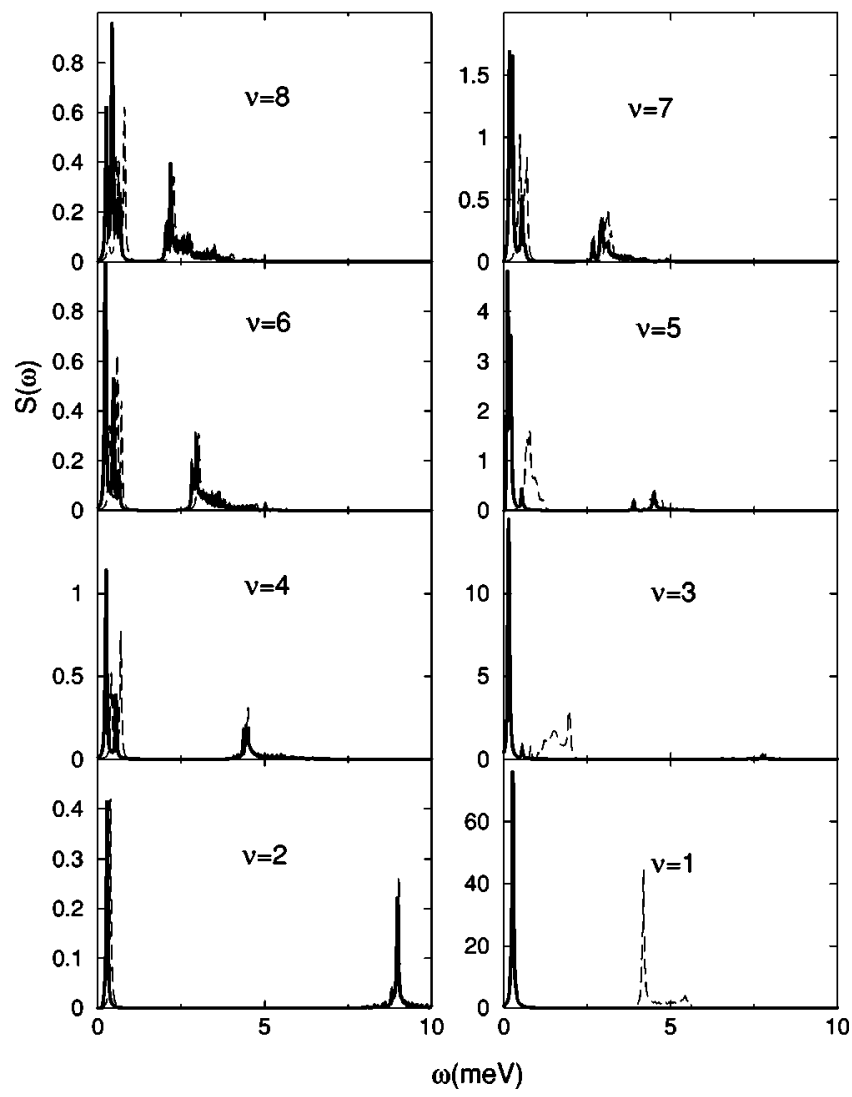

FIG. 4. Strength function corresponding to $x \sigma_{-}$(solid lines). The dashed lines represent the free strength function. $S(\omega)$ is in effective atomic units divided by $10^{5}$.

size effects distort the bands at the edge of the dot that is formed by sp states with high- $l$ values, it is clear that lowenergy spin-flip transitions involve sp states whose energy difference is precisely the energy difference between the $\left(M_{\max }, \uparrow \downarrow\right)$ bands. These excitations also occur in the bulk (2dEG). The role of the residual interaction is clearly visible in Fig. 4 comparing the free and TDCDFT strength functions at $\nu=5$ and at $\nu=3$, for example. One sees that the SPE energies have nothing to do with the low SDE energy. Thus, any free e-h model will be of little help to quantitatively analyze SDE's in partially polarized QD, the situation worsening the higher the polarization.

We present in Fig. 5 a more detailed picture of the spin excitation in the transverse channel at high $B$, showing the strength function of $x \sigma_{x}$ in the $2>\nu \geqslant 1$ region. As in previous figures, the dashed lines represent the free response. In this range of filling factors, which corresponds to $5.14 \mathrm{~T}$ $<B \leqslant 10.28 \mathrm{~T}$, we have found ${ }^{18}$ that the $2 S_{z}$ value steadily increases ${ }^{27}$ from zero to 210 , so the $x \sigma_{x}$ strength is essentially that of $x \sigma_{-}$already discussed. The interesting new feature in Fig. 5 is the structure of the high-frequency peaks. They are two orders of magnitude less intense than the lowenergy ones, which thus, exhaust most of the strength. Of the high-energy peaks, the higher ones are excited by $x \sigma_{-}$, and the lower ones by $x \sigma_{+}$(obviously, high-energy transitions caused by $x \sigma_{+}$are blocked only when the system is fully polarized). It can be seen that these high-energy peaks are

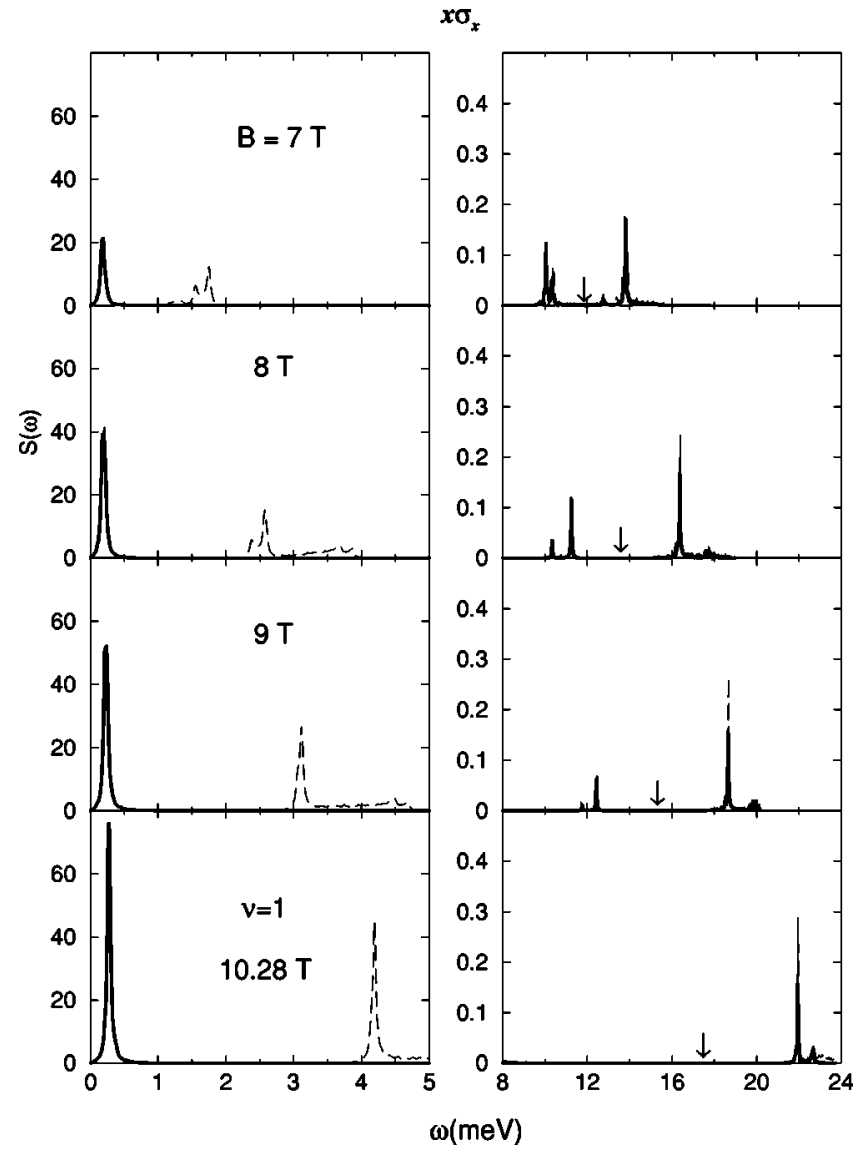

FIG. 5. Same as Fig. 2 for $2>\nu \geqslant 1$. The vertical arrows indicate the value of $\omega_{c}$.

little collective, as SPE's and SDE's are quite similar, and also that the centroid of the $x \sigma_{+}$and $x \sigma_{-}$peaks roughly follows the same evolution with $B$ as the cyclotron frequency $\omega_{c}=e B / m c$ does. The value of $\omega_{c}$ is indicated in Fig. 5 by vertical arrows.

When both high-energy peaks are clearly visible in the strength, as for example at $B=7 \mathrm{~T}$, their splitting is a quantitative measure of the spin-dependent exchange-correlation gap $W^{x c}$, and its measurement may be the spectroscopic complement to experimental gap determinations based on the temperature dependence of the conductivity. ${ }^{28}$ This is so because $W^{x c}+g^{*} \mu_{B} B / 2$ is directly related to the energy difference of the $(M, \uparrow)$ and $(M, \downarrow)$ bands around the Fermi level, see Eq. (7). As the sets of "parallel" bands $(M+1, \uparrow \downarrow)$ and $(M, \uparrow \downarrow)$ have the same "width" as Fig. 1 indicates, the splitting between the high-energy peaks would be twice the energy difference between the $\left(M_{\max }, \uparrow\right)$ and $\left(M_{\max }, \downarrow\right)$ bands. This is nicely confirmed in the $B=7$ and $9 \mathrm{~T}$ cases, for which an explicit comparison is possible (see Fig. 5 of Ref. 18). We recall that this comparison is meaningful because of the weak effect of the residual interaction on the high-energy peaks.

The longitudinal high-energy peak for these configurations lies ${ }^{16}$ at $\omega^{\text {lon }} \sim \omega_{c}$ (see also Fig. 7), and an expression similar to Eq. (3) can be written:

$$
\omega_{ \pm}^{t r} \sim \omega^{l o n} \pm 2 W^{x c} .
$$




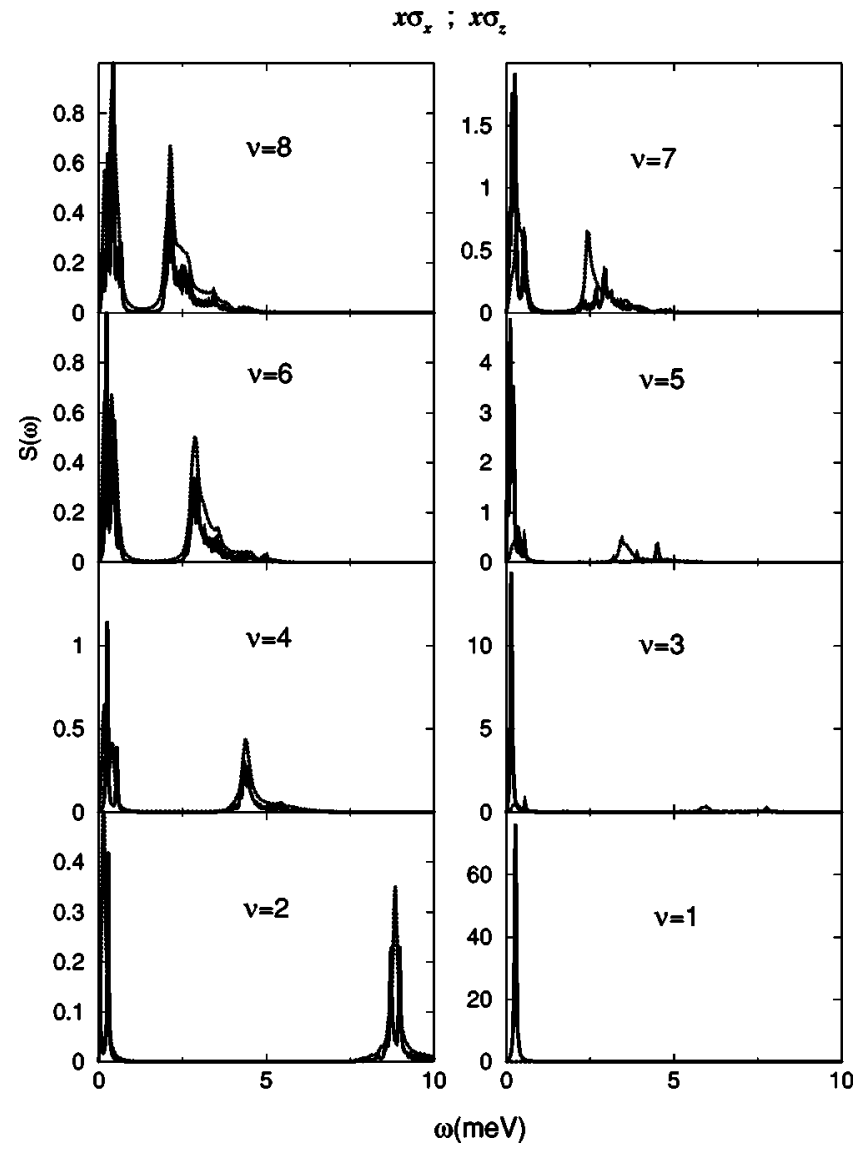

FIG. 6. Strength function corresponding to $x \sigma_{x}$ (solid line) and $x \sigma_{z}$ (dotted line). The strengths are in effective atomic units divided by $10^{5}$ in the transverse case, and by $5 \times 10^{4}$ in the longitudinal case to make it easier to distinguish them.

The validity of Eqs. (3) and (8) is a consequence of the weakness of the residual e-h interaction when they hold (notice that $g^{*}$ and $W^{x c}$ are negative).

Figure 6 shows the longitudinal $\left(x \sigma_{z}\right)$ and transverse $\left(x \sigma_{x}\right)$ dipole spin strength functions as dotted and solid lines, respectively. It can be seen that in ferromagnetic states, the strength is dominated by the transverse modes. One should also notice that for $\nu \geqslant 2$, i.e., low $B$, apart from some fine structure the longitudinal and transverse spin responses have their main peaks at quite similar energies.

The paramagnetic configuration at $\nu=2(B=5.14 \mathrm{~T})$ shows the interesting situation in which a zero spin gs sustains the simple result anticipated at the introduction: the transverse modes are just shifted by the Zeeman energy from the longitudinal ones. Interestingly too, the low energy $x \sigma_{+}$ mode has almost collapsed. This suggests the presence of instabilities in this particular transverse channel, similar to those found in the longitudinal spin channel in the $2 \geqslant \nu$ $>1$ region. $^{16}$

When the system is fully polarized, the longitudinal spin and charge density strengths trivially coincide and of the spin modes, only the transverse one has a sense. ${ }^{21,22,16}$ The connection between the peak and Zeeman energies will be discussed in the next subsection.

The energies of the more intense, high-energy peaks appearing in the $x \sigma_{+}$and the $x \sigma_{-}$strength functions are shown in Fig. 7 as a function of $B$. The cyclotron frequency

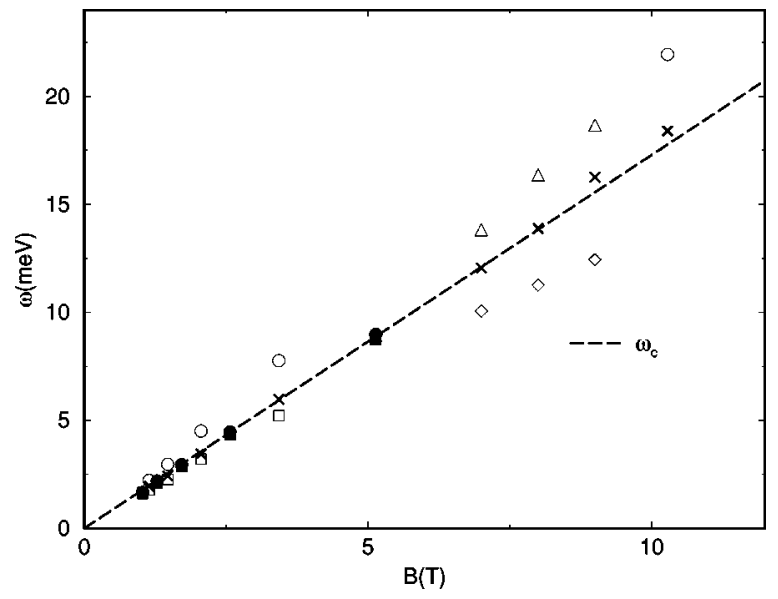

FIG. 7. Energies of the more intense high-energy peaks excited by $x \sigma_{+}$(squares) and $x \sigma_{-}$(circles) as a function of $B$. Solid symbols correspond to even filling-factor values, and empty symbols to odd filling-factor values from $\nu=10(B=1.03 \mathrm{~T})$ to $\nu=1(B$ $=10.28 \mathrm{~T}$ ). Also drawn are the energies of the high-energy longitudinal spin peaks (crosses). The triangles and diamonds correspond to $B=7,8$, and $9 \mathrm{~T}$.

is also represented. Solid symbols correspond to even filling factor values, and open symbols to odd filling factor values from $\nu=10(B=1.03 \mathrm{~T})$ to $\nu=1 \quad(B=10.28 \mathrm{~T})$. Also drawn are the values of the high-energy longitudinal spin peaks ${ }^{16}$ (crosses). The triangles and diamonds correspond to the $B=7,8$, and 9 T peaks in the right panels of Fig. 5 .

In agreement with the preceding discussion, it is seen that for even filling factors the energies of the transverse SDE are $\sim \omega_{c} \pm g^{*} \mu_{B} B$, thus, close to $\omega_{c}$, whereas for odd filling factors they are well apart from $\omega_{c}$ by the large spindependent potential $W^{x c}$. In all cases, these peaks correspond to bulk modes involving interband e-h transitions made of $\mathrm{sp}$ states each one belonging to a Landau band with different index $M$.

Similarly, Fig. 8 collects the energies of the more intense, low-energy peaks appearing in the $x \sigma_{+}$(top panel) and of $x \sigma_{-}$(bottom panel) strength functions. Solid and open symbols have the same meaning as in Fig. 7. The Zeeman energy $E_{z}=-g^{*} \mu_{B} B>0$ is also represented. To emphasize the energy staggering, consecutive $\nu$ points have been connected by a thin line. As we have already discussed, these modes are spin edge modes for paramagnetic gs's, and spin bulk modes for ferromagnetic gs's.

The SDE corresponding to $x \sigma_{-}$is the more interesting one. It is the only transverse spin mode that appears at high magnetizations, since the one generated by $x \sigma_{+}$is Pauli blocked. For ferromagnetic gs's, this is an undamped excitation since it is well apart from the SPE's (see Fig. 4). Notice that the transverse SDE energy approaches the Zeeman energy as $B$ increases. At full polarization $(\nu=1)$, the energy is close to $E_{z}$, but not equal to it. We shall come back to this point in the following.

\section{B. Sum rules}

Further insight onto the strength functions can be obtained from the evaluation of sum rules, which are their energy moments. Some of these moments are easy to obtain, model 


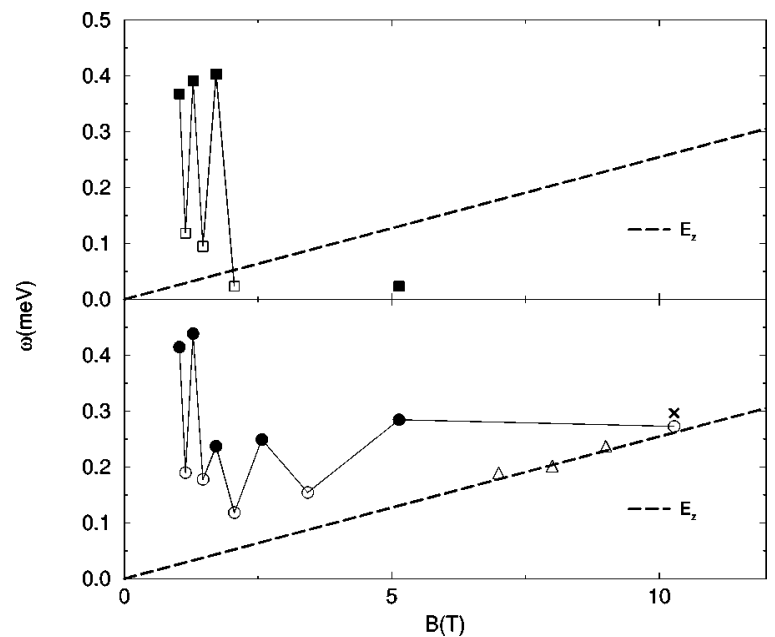

FIG. 8. Bottom panel: Energies of the more intense low-energy peaks excited by $x \sigma_{-}$as a function of $B$ for the same configurations as in Fig. 7. Also shown is the Zeeman energy (dashed line). The cross at $\nu=1$ is the SDE value obtained from TDCDFT when the sp valence space is limited to the $M=0$ bands. Top panel: Same as bottom panel for $x \sigma_{+}$. Some configurations are absent due to Pauli blocking.

independent quantities. We are interested here in sum rules for non-Hermitian excitation operators as the $F_{ \pm}$ones defined in Eq. (1). These sum rules have been extensively discussed in Ref. 29.

We consider the usual Pauli Hamiltonian $H$ describing an $N$ electron QD submitted to a constant magnetic field in the $z$ direction (see for example Ref. 30) and define the following sum rules $(\mathrm{SR})$ :

$$
\begin{gathered}
S_{0} \equiv S_{0}^{-}-S_{0}^{+} \equiv \sum_{n}\left|\left\langle n\left|F_{-}\right| 0\right\rangle\right|^{2}-\sum_{n}\left|\left\langle n\left|F_{+}\right| 0\right\rangle\right|^{2}, \\
S_{1} \equiv S_{1}^{-}+S_{1}^{+} \equiv \sum_{n} \omega_{n 0}\left|\left\langle n\left|F_{-}\right| 0\right\rangle\right|^{2}+\sum_{n} \omega_{n 0}\left|\left\langle n\left|F_{+}\right| 0\right\rangle\right|^{2},
\end{gathered}
$$

where $|0\rangle$ is the gs of the system and $|n\rangle$ is an excited state with excitation energy $\omega_{n 0}$. Using closure, it is easy to check that

$$
\begin{gathered}
S_{0}=\left\langle 0\left|\left[F_{+}, F_{-}\right]\right| 0\right\rangle, \\
S_{1}=\langle 0|\left[F_{+},\left[H, F_{-}\right]|0\rangle .\right.
\end{gathered}
$$

An explicit evaluation of $S_{0}$ and $S_{1}$ only valid for the dipole $x$ (or $y$ ) and plane-wave operators (the general case will be given elsewhere) yields

$$
S_{0}=\int d \vec{r}|f(\vec{r})|^{2} m_{0}(\vec{r}),
$$

$$
S_{1}=\frac{1}{2} \int d \vec{r}|\nabla f(\vec{r})|^{2} \rho_{0}(\vec{r})-g^{*} \mu_{B} B \int d \vec{r}|f(\vec{r})|^{2} m_{0}(\vec{r}),
$$

where $\rho_{0}(\vec{r})$ is the gs density of the dot, and $m_{0}(\vec{r})$ $\equiv \rho_{0}^{\uparrow}(\vec{r})-\rho_{0}^{\downarrow}(\vec{r})$ is the gs local spin magnetization. These sum rules are relating properties of the exact spectrum of the Pauli Hamiltonian to properties of its exact gs.
$S_{0}$ and $S_{1}$ are also fulfilled within TDDFT. Indeed, it can be proved using the techniques discussed in Ref. 29, that the TDDFT spectrum is such that one can obtain them with TDDFT accuracy from Eq. (11) using the KS gs. Not all approximation schemes fulfill these sum rules. Independent particle spectra such as those obtained from HF, KS, or Hartree approximations violate $S_{1}$. This means that one would not obtain the second of Eq. (11) using in the second of Eq. (10) the corresponding one-body Hamiltonian. For example, the KS spectrum we use to build the SPE's and $\chi^{0}$ correlation function yields

$$
\begin{aligned}
S_{1}^{K S}= & \frac{1}{2} \int d \vec{r}|\nabla f(\vec{r})|^{2} \rho_{0}(\vec{r}) \\
& -\int d \vec{r}\left[g^{*} \mu_{B} B+2 W^{x c}(\vec{r})\right]|f(\vec{r})|^{2} m_{0}(\vec{r}) .
\end{aligned}
$$

Within TDDFT, the effect of the e-h induced interaction is crucial in restoring $S_{1}$ : it exactly cancels the $2 W^{x c}$ contribution in Eq. (12). We want to point out in passing that in the density channel, the induced interaction is also responsible for the fulfillment of Kohn's theorem, which would be violated otherwise.

When the dot has a large gs spin magnetization, the $x \sigma_{+}$ term in Eq. (9) contributes very little to $S_{0}$ and $S_{1}$. Thus, one is left with the $x \sigma_{-}$contribution, which is concentrated in a narrow energy region (see right panels in Fig. 4). Under these conditions, it is a fair approximation to identify $\bar{\omega}$ $\equiv S_{1} / S_{0}$ with the mean energy of the peak displayed in the $x \sigma_{-}$strength function. To proceed further, let us consider that the dot is fully polarized, i.e., $m_{0}(\vec{r})=\rho_{0}(\vec{r})$. Taking $f(\vec{r})=x$, one gets

$$
S_{0}=S_{0}^{-}=\frac{N}{2}\left\langle r^{2}\right\rangle
$$

$$
S_{1}=S_{1}^{-}=\frac{N}{2}-\frac{N}{2} g * \mu_{B} B\left\langle r^{2}\right\rangle,
$$

where $\left\langle r^{2}\right\rangle$ is the root-mean-square radius of the dot

$$
\left\langle r^{2}\right\rangle \equiv \frac{1}{N} \int d \vec{r} r^{2} \rho_{0}(\vec{r})
$$

Thus,

$$
\bar{\omega}=\frac{S_{1}}{S_{0}}=-g^{*} \mu_{B} B+\frac{1}{\left\langle r^{2}\right\rangle} .
$$

At $\nu=1$, taking for $\rho_{0}(\vec{r})$ that of the maximum density droplet (MDD) state, ${ }^{31}$ which is a good approximation for large dots, we have

$$
\bar{\omega}=\frac{S_{1}}{S_{0}}=-g^{*} \mu_{B} B+\frac{\omega_{c}}{N+1} .
$$

Expressions (15) or (16) are the SR estimates of the transverse dipole SDE at large spin magnetization. Using again the $\operatorname{MDD}\left\langle r^{2}\right\rangle$ value, we get $S_{0}^{-} \sim N(N+1) / 2 \omega_{c}$. This 
shows that for high-spin magnetizations, the squared $x \sigma_{-}$ matrix elements in the SDE's are a factor $\sim N$ stronger than the squared $x$ matrix elements in the CDE's, whose strength is $\sim N / 2 \omega_{c} .{ }^{30}$

For GaAs, $\omega_{c} /\left|g * \mu_{B} B\right|=2 /\left|g * m^{*}\right| \sim 68$. We thus have $\bar{\omega} \sim 0.35 \mathrm{meV}$ for the $N=210$ dot at $\nu=1$, whereas the peak energy is $E_{\text {peak }} \sim 0.27 \mathrm{meV}$ and $E_{z} \sim 0.26 \mathrm{meV}$. In the limit of a very large dot, $\bar{\omega}$ becomes $E_{z}$. This is the analogous of the $2 \mathrm{DEG}$ result ${ }^{21,22}$ for the spin-wave dispersion relation at $q=0$ (Larmor's theorem).

So far, we have discussed the response to the dipole $L$ $=1$ operator. It is straightforward to consider the response to a general $L$ mode operator of the kind $f(\vec{r}) \sim r^{L} e^{ \pm \imath L \theta}$. These fields are relevant to study spin and charge-density modes with well-defined angular momentum. Moreover, in Raman experiments one may face a situation in which the in-plane transferred linear momentum $q$ is small enough, so that $q R_{d o t} \ll 1$ and the plane wave operator $e^{l \vec{q} \vec{r}}$ involved in the excitation process can be expanded into $r^{L} e^{ \pm \imath L \theta}$ multipoles, each of them probing a well defined angular momentum CDE or SDE. ${ }^{4}$ When $q$ cannot be considered as small, ${ }^{2}$ the TDDFT response may still be worked out, fixing $q$ and adding the responses to $f(\vec{r})=J_{L}(q r) e^{ \pm \imath L \theta}$, because $e^{\imath \vec{q} \vec{r}}$ $=\Sigma_{L} l^{L} J_{L}(q r) e^{\imath L \theta}$. The number of terms in the expansion may be large depending on the $q$ value, but in principle the method is of direct applicability.

We finish this subsection discussing the SR for $f(\vec{r})$ $=e^{i \vec{q} \vec{r}}$ in the more interesting case of full polarization. Since $|f(\vec{r})|^{2}=1$ and $|\nabla f(\vec{r})|^{2}=q^{2}$, we get from Eq. (11)

$$
\begin{gathered}
S_{0}=N, \\
S_{1}=\frac{1}{2} q^{2} N-g^{*} \mu_{B} B N .
\end{gathered}
$$

The same equations hold for the 2DEG substituting $N$ by the electron density. In either case,

$$
\bar{\omega}=-g^{*} \mu_{B} B+\frac{1}{2} q^{2} .
$$

We want to emphasize that Eqs. (17) and (18) are exact, model independent and valid for any $q$ value. Only the applicability of the Pauli Hamiltonian to describe this physical situation has been taken for granted.

At first glance, Eq. (18) is in contradiction with the spinwave dispersion relation of Refs. 21 and 22, whose $q$ dependent term has an electron-electron energy dependence instead of the $\frac{1}{2}$ factor. The difference stems from the $\mathrm{sp}$ valence space, which is different in both calculations. Indeed, the sum rule result Eq. (18) takes into account all possible intraband and interband $\mathrm{sp}$ excitations induced by the operator $e^{l \vec{q} \vec{r}} \sigma_{-}$. Thus, $\bar{\omega}$ is an average of the low- and high-energy peaks. In Refs. 21 and 22, the valence space was restricted to the filled $(0, \uparrow)$ and empty $(0, \downarrow)$ bands to specifically address the low-energy mode. Their result can be exactly recovered in the SR approach if one uses the same valence space and accordingly, the projection of $e^{i \vec{q} \vec{r}} \sigma_{-}$ onto the $M=0$ space. $^{32}$ This is the so-called single-mode approximation, ${ }^{33}$ equivalent to the approach of Refs. 21 and 22 in the fully polarized case.

It is easy to seize the effect of the $M>0$ bands on the low-energy collective mode within TDCDFT. It suffices to compute the response limiting the sp valence space to the $M=0$ bands. In this case, only the low-energy peak appears in the $x \sigma_{-}$strength function, and its energy $\sim 0.30 \mathrm{meV}$ is denoted by a cross in the bottom panel of Fig. 8. The inclusion of the high-energy bands changes the energy of the transverse SDE in $\sim 10 \%$. We have also determined that the high-energy peaks in the $x \sigma_{-}$strength contribute around $20 \%$ to $S_{1}$.

\section{SUMMARY}

In this paper, we have thoroughly studied the transverse dipole spin response in quantum dots. Together with our previous works, ${ }^{15,19,16}$ they provide a detailed account of the applicability of time-dependent density-functional theory to the description of the dipole response of QD in the charge and spin channels. Although microscopic descriptions of similar complexity exist since some time ago for the charge density modes in medium size QD ${ }^{34,35,23}$ the spin density modes in QD had not been previously addressed. Besides, we have been able to apply the theory to rather large dots, similar in size to those investigated in present experiments. ${ }^{12,1-4}$ A thorough comparison with recent experiments ${ }^{4}$ requires to extend the method to arbitrary multipolarities and to take into account the on-plane transferred momentum. This paper is now in progress and will be reported elsewhere. $^{36}$

Among SDE's, the transverse ones are especially relevant; in the longitudinal channel, the residual interaction is fairly weak, and the SDE's are Landau damped as they are close to the SPE's (actually, the same happens with the transverse modes at low $B$ ). In the transverse channel, when the dot has a sizeable magnetization the position of SDE's is shifted away from the SPE's by exchange-correlation vertex corrections arising from electron-electron interactions. As a consequence, a very collective, dispersionless SDE emerges. At large spin magnetization, Pauli's principle plays a prominent role, blocking the $x \sigma_{+}$component of the transverse strength function, which becomes simpler.

The possibility of carrying the calculations in a large dot displaying several integer quantum Hall gs's has permitted us to disclose the sensitivity of the transverse response to the applied $B$, which appears as a strong energy oscillation with $B$, and a nearly collapsed low energy $x \sigma_{+}$mode at the $\nu$ $=2$ paramagnetic gs. The energy oscillations are also consequence of the different strength of vertex corrections in ferromagnetic and paramagnetic gs's.

We have also derived two model-independent sum rules that, on the one hand, can be used as a control for the analysis of the experimental data, and on the other hand, have allowed us to relate our results at full magnetization to previous works on $2 \mathrm{DEG}$.

\section{ACKNOWLEDGMENTS}

This work has been performed under Grants Nos. PB951249 and PB95-0492 from DGICYT, and Grant No. 1998SGR00011 from Generalitat de Catalunya. A.E. and M.B. (Ref. PR1997-0174) acknowledge support from the DGES (Spain). 
* Permanent address: Departament d'Estructura i Constituents de la Matèria, Facultat de Física, Universitat de Barcelona, E-08028 Barcelona, Spain.

${ }^{1}$ R. Strenz, U. Bockelmann, F. Hirler, G. Abstreiter, G. Böhm, and G. Weimann, Phys. Rev. Lett. 73, 3022 (1994).

${ }^{2}$ D. J. Lockwood, P. Hawrylak, P. D. Wang, C. M. Sotomayor Torres, A. Pinczuk, and B. S. Dennis, Phys. Rev. Lett. 77, 354 (1996).

${ }^{3}$ C. Schüller, G. Biese, K. Keller, C. Steinebach, D. Heitmann, P. Grambow, and K. Eberl, Phys. Rev. B 54, R17 304 (1996).

${ }^{4}$ C. Schüller, K. Keller, G. Biese, E. Ulrichs, L. Rolf, C. Steinebach, and D. Heitmann, Phys. Rev. Lett. 80, 2673 (1998).

${ }^{5}$ A. Pinczuk, S. Schmitt-Rink, G. Danan, J. P. Valladares, L. N. Pfeiffer, and K. W. West, Phys. Rev. Lett. 63, 1633 (1989).

${ }^{6}$ A. Schmeller, A. R. Goñi, A. Pinczuk, J. S. Weiner, J. M. Calleja, B. S. Dennis, L. N. Pfeiffer, and K. W. West, Phys. Rev. B 49, 14778 (1994).

${ }^{7}$ V. Pellegrini, A. Pinczuk, B. S. Dennis, A. S. Plaut, L. N. Pfeiffer, and K. W. West, Phys. Rev. Lett. 78, 310 (1997).

${ }^{8}$ A. S. Plaut, A. Pinczuk, P. I. Tamborenea, B. S. Dennis, L. N. Pfeiffer, and K. W. West, Phys. Rev. B 55, 9282 (1997).

${ }^{9}$ A. Pinczuk and G. Abstreiter, Light Scattering in Solids V, edited by M. Cardona and G. Guntherodt, Topics in Applied Physics Vol. 66 (Springer-Verlag, Berlin, 1989) p. 153.

${ }^{10}$ A. Pinczuk, D. Heiman, S. Schmitt-Rink, C. Kallin, B. S. Dennis, L. N. Pfeiffer, and K. W. West, in Light Scattering in Semiconductor Structures and Superlattices, edited by D. J. Lockwood and J. F. Young (Plenum Press, New York, 1991), p. 571.

${ }^{11}$ Ch. Sikorski and U. Merkt, Phys. Rev. Lett. 62, 2164 (1989).

${ }^{12}$ T. Demel, D. Heitmann, P. Grambow, and K. Ploog, Phys. Rev. Lett. 64, 788 (1990).

${ }^{13}$ S. Katayama and T. Ando, J. Phys. Soc. Jpn. 54, 1615 (1985).

${ }^{14}$ M. S. C. Luo, S. L. Chuang, S. Schmitt-Rink, and A. Pinczuk, Phys. Rev. B 48, 11086 (1993).

${ }^{15}$ Ll. Serra and E. Lipparini, Europhys. Lett. 40, 667 (1997).
${ }^{16}$ L1. Serra, M. Barranco, A. Emperador, M. Pi, and E. Lipparini, Phys. Rev. B 59, 15290 (1999).

${ }^{17}$ M. Ferconi and G. Vignale, Phys. Rev. B 50, 14722 (1994).

${ }^{18}$ M. Pi, M. Barranco, A. Emperador, E. Lipparini, and Ll. Serra, Phys. Rev. B 57, 14783 (1998).

${ }^{19}$ E. Lipparini and Ll. Serra, Phys. Rev. B 57, R6830 (1998).

${ }^{20}$ G. Vignale and M. Rasolt, Phys. Rev. B 37, 10685 (1988).

${ }^{21}$ C. Kallin and B. I. Halperin, Phys. Rev. B 30, 5655 (1984).

${ }^{22}$ A. H. MacDonald, J. Phys. C 18, 1003 (1985).

${ }^{23}$ V. Gudmundsson and J. J. Palacios, Phys. Rev. B 52, 11266 (1995).

${ }^{24}$ S. L. Sondhi, A. Karlhede, S. A. Kivelson, and E. H. Rezayi, Phys. Rev. B 47, 16419 (1993).

${ }^{25}$ L. Brey, N. F. Johnson, and B. I. Halperin, Phys. Rev. B 40, 10 647 (1989)

${ }^{26}$ P. A. Maksym and T. Chakraborty, Phys. Rev. Lett. 65, 108 (1990).

27 This is at variance with what happens for a small QD in a parabolic potential, for which $2 S_{z}$ fluctuates in the same $\nu$ region.

${ }^{28}$ A. Usher, R. J. Nicholas, J. J. Harris, and C. T. Foxon, Phys. Rev. B 41, 1129 (1990).

${ }^{29}$ E. Lipparini and S. Stringari, Phys. Rep. 175, 103 (1989).

${ }^{30}$ E. Lipparini, N. Barberan, M. Barranco, M. Pi, and Ll. Serra, Phys. Rev. B 56, 12375 (1997).

${ }^{31}$ A.H. MacDonald, S.R. Eric Yang, and M.D. Johnson, Aust. J. Phys. 46, 345 (1993).

${ }^{32}$ S. M. Girvin and T. Jach, Phys. Rev. B 29, 5617 (1984).

${ }^{33}$ S. M. Girvin, A. M. MacDonald, and P. M. Platzman, Phys. Rev. B 33, 2481 (1986).

${ }^{34}$ D. A. Broido, K. Kempa, and P. Bakshi, Phys. Rev. B 42, 11400 (1990).

${ }^{35}$ V. Gudmundsson and R.R. Gerhardts, Phys. Rev. B 43, 12098 (1991).

${ }^{36}$ M. Barranco, L. Colletti, A. Emperador, E. Lipparini, M. Pi, and Ll. Serra, cond-mat/9907321 (unpublished). 